University of Wollongong

Research Online

Faculty of Engineering - Papers (Archive)

Faculty of Engineering and Information

Sciences

April 1992

\title{
Hole-quasiparticle resonant polaron coupling in quantum wells containing high densities of free carriers
}

Philip E. Simmonds

University of Wollongong, simmonds@uow.edu.au

M. S. Skolnick

University of Sheffield, UK

T. A. Fisher

University of Wollongong

K. J. Nash

Royal Signals and Radar Establishment, UK

R. S. Smith

GEC Hirst Research Centre, UK

Follow this and additional works at: https://ro.uow.edu.au/engpapers

Part of the Engineering Commons

https://ro.uow.edu.au/engpapers/272

\section{Recommended Citation}

Simmonds, Philip E.; Skolnick, M. S.; Fisher, T. A.; Nash, K. J.; and Smith, R. S.: Hole-quasiparticle resonant polaron coupling in quantum wells containing high densities of free carriers 1992.

https://ro.uow.edu.au/engpapers/272

Research Online is the open access institutional repository for the University of Wollongong. For further information contact the UOW Library: research-pubs@uow.edu.au 


\title{
Hole-quasiparticle resonant polaron coupling in quantum wells containing high densities of free carriers
}

\author{
P. E. Simmonds \\ Department of Physics, University of Wollongong, Wollongong, NSW 2500, Australia \\ M. S. Skolnick \\ Department of Physics, University of Sheffield, Sheffield S3 7RH, United Kingdom \\ T. A. Fisher \\ Department of Physics, University of Wollongong, Wollongong, NSW 2500, Australia
}

K. J. Nash

Royal Signals and Radar Establishment, St. Andrew's Road, Malvern, Worcestershire, WRI4 3PS, United Kingdom

R. S. Smith

GEC Hirst Research Centre, Wembley, Middlesex, HA9 7PP, United Kingdom

(Received 10 October 1991)

\begin{abstract}
Strong resonant polaron coupling phenomena in the magnetophotoluminescence (magneto-PL) spectra of modulation-doped quantum wells are reported. The polaron interactions arise between hole quasiparticles in otherwise filled electron Landau levels, and LO phonons of the quantum-well material. This type of many-body polaron coupling phenomenon is expected to be a universal feature of the magneto-PL spectra of quantum wells with electron Fermi energy greater than the LO-phonon energy of the well material.
\end{abstract}

New resonant polaron coupling (RPC) phenomena in the magneto-optical spectra of quantum wells (QW's) containing high densities of free carriers are reported. The phenomena have a many-body character, since the resonances arise from the Fröhlich interaction between hole quasiparticles in otherwise filled electron Landau levels (LL's) and the LO-phonon modes of the QW material. It is shown that a common requirement for the observation of RPC in the magnetophotoluminescence (magneto-PL) of QW's is that the carrier density should be of sufficient magnitude that the electron Fermi energy is of the order of, or greater than, the LO-phonon energy of the QW material.

RPC has been much studied in both three-dimensional ${ }^{1,2}$ (3D) and quasi-2D (Refs. 3-6) systems, principally in cyclotron resonance (CR) experiments. ${ }^{7}$ In such cases, the RPC is usually observed as a strong cyclotron mass enhancement, the result of electron-LO-phonon interactions which lead to pronounced anticrossing between unoccupied LL's (LL index $N_{e}>0$ ) and the $N_{e}=0$ plus one-phonon state. ${ }^{1,8-10}$ In the present work, the resonant coupling is observed as an interaction between LO-phonon satellites of recombination involving electrons in upper $\mathrm{LL}$ states ( $L L$ index $N_{e}>2$ ) and PL transitions from the $N_{e}=0$ LL." Large values for the resonant polaron interaction strength, in comparison with $\mathrm{CR}$ experiments, are observed. This is attributed to the absence of LL occupation effects ${ }^{8-10}$ for the hole quasiparticles.

The magneto-PL experiments were carried out on a series of asymmetric, modulation-doped $\mathrm{Al}_{y} \mathrm{Ga}_{1-y} \mathrm{As}$ $\operatorname{In}_{x} \mathrm{Ga}_{1-x} \mathrm{As}-\mathrm{GaAs} \quad(y \approx 0.23, \quad x \approx 0.1)$ strained-layer
QW's with high $n_{s}$ values ranging from $(0.8-1.6) \times 10^{12}$ $\mathrm{cm}^{-2}$ and well widths of 150-250 $\AA$. Zero-field results are discussed elsewhere. ${ }^{12.13}$ The particular sample investigated in detail has a QW width of $150 \AA$ and $n_{s}=1.6 \times 10^{12} \mathrm{~cm}^{-2}\left(E_{F}=56 \mathrm{meV}\right)$, determined from Shubnikov-de Haas measurements. Magneto-PL measurements were carried out at $4.2 \mathrm{~K}$ in magnetic fields $(B)$ up to $10 \mathrm{~T}$.

The PL spectrum at $B=0$ is shown in Fig. 1(a). It is composed of two bands, $E_{11}$ and $E_{21}$, arising from recombination of electrons in the $n=1$ and $n=2$ subbands, respectively, with photocreated holes thermalized in the lowest heavy-hole subband close to $k=0$. The spectra extend from the bottom of the $n=1$ subband at $E_{1}$ up to an energy corresponding to $E_{F}$. The $\Delta k_{\perp}=0$ in-plane wavevector selection rule is broken by disorder (or hole localization) effects ${ }^{14,15}$ giving rise to the $E_{11}$ transitions, observed with strongly decreasing strength above the $n=1$ subband edge.

$E_{21}$ transitions are seen since the $E_{F}$ of $56( \pm 1) \mathrm{meV}$ is very close to the $E_{2}-E_{1}$ subband separation $(56 \pm 1$ $\mathrm{meV}$ ), with an upper limit to the $n=2$ population of $5 \times 10^{10} \mathrm{~cm}^{-2}$ deduced from the absence of any LL splitting of the $E_{21}$ PL. The $E_{21}$ peak is $\sim 3$ times stronger than $E_{11}$ principally because of the larger overlap of $E_{2}$ electrons with the photocreated holes than that for $E_{1}$ electrons. ${ }^{13}$

In magnetic field the $E_{11}$ PL breaks up into a series of LL peaks, labeled $\left(N_{e}, 0\right)_{1}$, as shown in Figs. $1(\mathrm{~b})-1(\mathrm{e})$, arising from recombination of electrons in LL's (index $N_{e}$ ) with holes in the lowest valence-band LL. The 


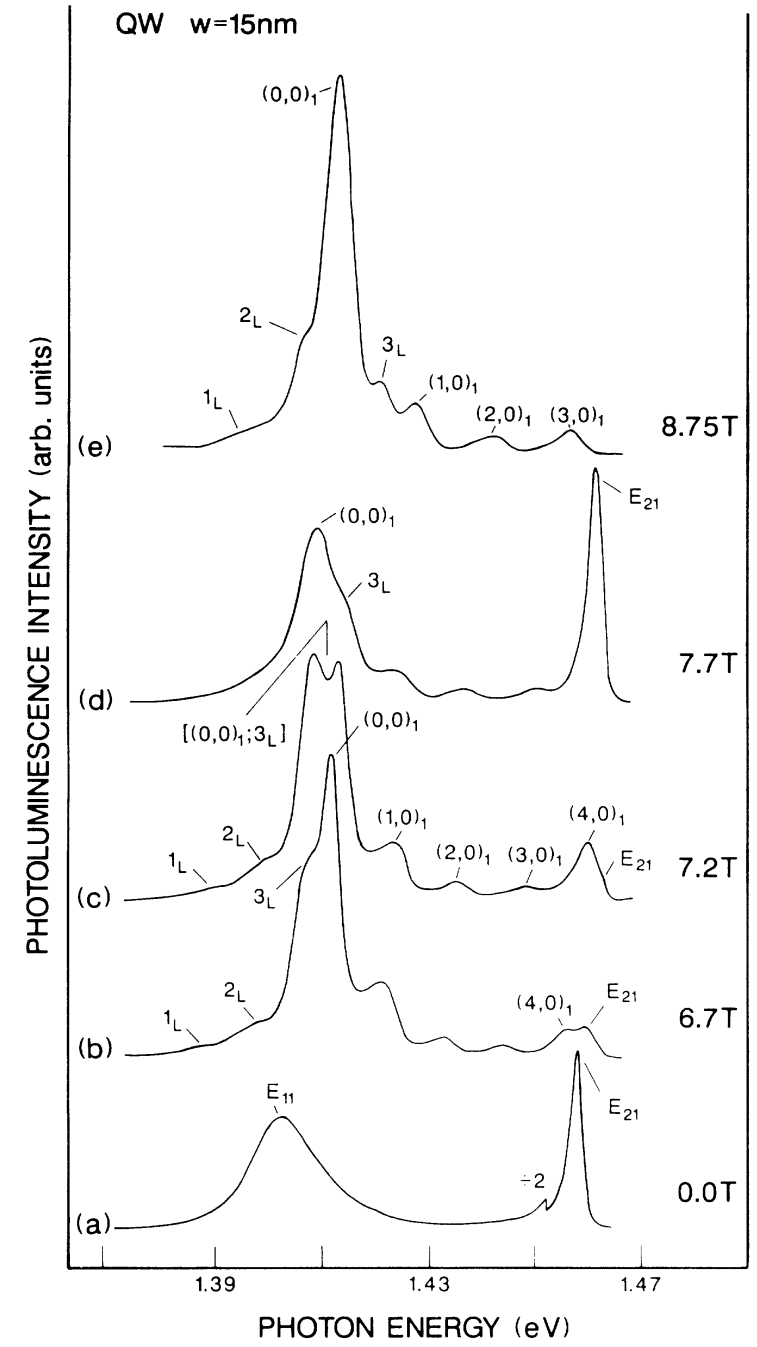

FIG. 1. PL spectra as a function of magnetic field. (a) $n=1$ and $n=2$ subband $E_{11}$ and $E_{21}$ recombination is observed at $B=0$. In (b)-(e) splitting into LLs $\left(N_{c}, 0\right)$, is observed. LOphonon satellites $N_{l}$. of $\left(N_{e}, 0\right)$, are observed with resonant enhancement of intensity as they approach $(0,0)$,

$\Delta N=0$ selection rule is broken by disorder for the same reason that nonvertical transitions occur at zero field. ${ }^{14}$ The energy spacings of the higher LL transitions $\left(N_{e}>1\right)$ are given by the electron cyclotron energy $\hbar \omega_{c}$, where $\omega_{c}=e B / m_{e}$ with $m_{e}=0.069 m_{0}{ }^{15}$ The $E_{21}$ peak shows strong oscillations in intensity with field [see Figs. 1(b)-1(e)], of very similar form to those reported by Chen and co-workers. ${ }^{16,17}$ The interpretation of these oscillations will be discussed in detail elsewhere.

For the present paper, the most important information is contained in the low-energy peaks labeled $N_{L}=1_{L}, 2_{L}$, $3_{L}, \ldots$ These $N_{L}$ satellites arise very close to the GaAs LO-phonon energy of $\hbar \omega_{\mathrm{LO}}=36.7 \mathrm{meV}$ below the corresponding $\left(N_{e}, 0\right)_{1}$ transitions. The energy of the GaAslike mode of the $\mathrm{QW}$, which contains only $9 \%$ In, will correspond closely to this value. The PL transition energies are plotted as a function of field in Fig. 2. The $\left(N_{e}, 0\right)_{1}$ Landau fan is reproduced by the $N_{L}$ LO-phonon satellite

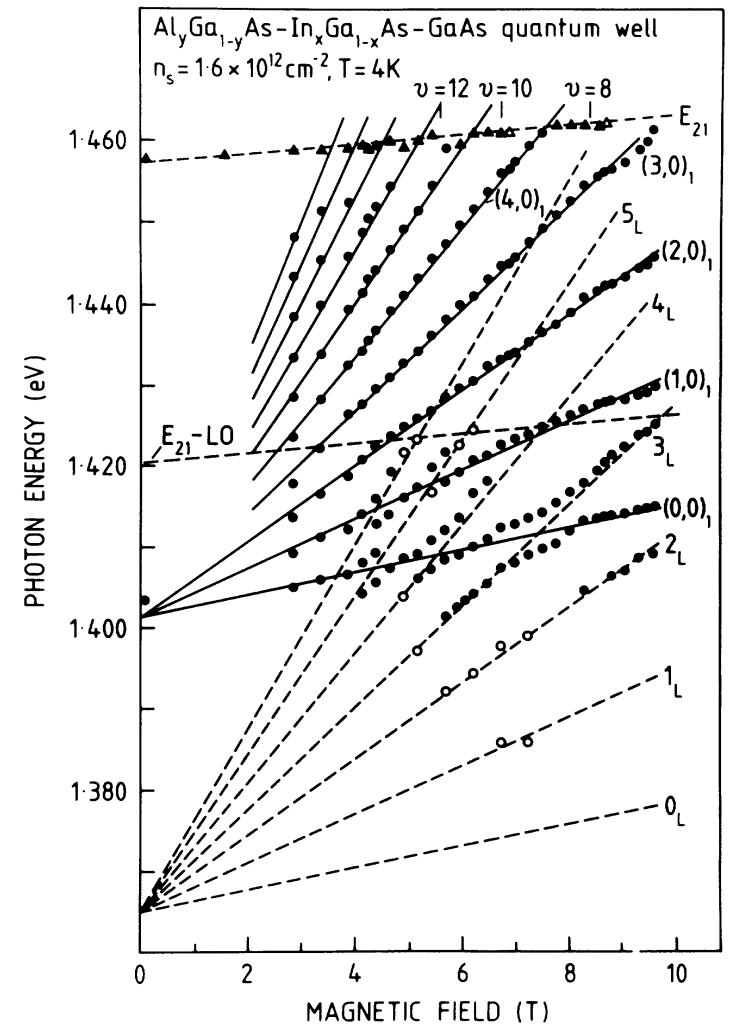

FIG. 2. PL energies of transitions in Fig. 1 as a function of magnetic field. The $N_{t}$, transitions are one LO-phonon energy below the $\left(N_{c}, 0\right)$, peaks. The resonant coupling between the $3 t$. and $4_{l}$. satellites and $(0,0)$, is clearly visible. Triangles denote $E_{21}$ transitions and solid circles denote $\left(N_{c}, 0\right)_{1}, N_{l}$ transitions. Open symbols denote weak transitions. No features at $E_{21}$ LO are observed.

lines displaced to lower energy by $\hbar \omega_{\text {LO }}$. Very marked resonant anticrossing between the lower-lying $\left(N_{e}, 0\right)_{\text {, }}$ transitions and the $N_{L}$ lines is observed around fields of $4.4,5.5$, and 7.3 $\mathrm{T}$ given by the "magnetophonon" resonance condition $\Delta N_{\mathrm{c}} \hbar \omega_{\mathrm{c}}=\hbar \omega_{\mathrm{LO}}{ }^{18} \Delta N_{\mathrm{c}}$ is the difference in Landau index between the parent LL of the $N_{l}$ satellite and the $N_{c}$, level with which resonance occurs.

In the region of resonance, clear exchange of oscillator strength occurs between $N_{L}$ and $\left(N_{e}, 0\right)_{1}$, as seen in Figs. 1(b)-1(e). Away from resonance the satellite $3_{L}$ for example, has $<1 \%$ of the $(0,0)_{1}$ intensity. As $3_{L}$ approaches $(0,0)$, from lower energy its intensity increases until at resonance where the two transitions are at their minimum separation $\left[\Delta E=4.4 \mathrm{meV}\right.$ for $3_{L}$ with $\left.(0,0)_{1}\right]$ the intensities are approximately equal [Figs. 1(c) and 4]. Beyond this field the lower component is pinned to the energy of the unperturbed $(0,0)$, transition while the 3 . character is transferred to the upper component and its intensity decreases. Near resonance, the $N_{l}$ satellite intensities far exceed those of the weak parent $\left(N_{\epsilon}, 0\right)$, zerophonon transitions. The pinning behavior and exchange of oscillator strength are characteristic of hybridizing states in general and specifically of the coupled LL-LOphonon states involved in RPC. ${ }^{8}$ 
Almost identical resonance behavior is found in a similar sample which has slightly lower $n_{s}\left(1.45 \times 10^{12} \mathrm{~cm}^{-2}\right.$, $E_{F}=48 \mathrm{meV}$ ) and only one occupied electron subband. Two further asymmetric QW's of widths 200 and $250 \AA$ with $E_{F}$ between 35 and $40 \mathrm{meV}$ also gave qualitatively similar resonances. In addition, strong resonant enhancement of LO satellites has been seen in an (In, Ga)As-InP QW $\left(n_{s}=10^{12} \mathrm{~cm}^{-2}\right.$, width $\left.100 \AA\right) .^{19}$

Unlike conventional CR measurements, in the present experiments both the parent $\mathrm{LL}$ of the $N_{L}$ satellite and the $N_{e}$ level involved in the resonance are, generally, fully occupied. The resonant coupling occurs in the final states of the PL transitions between quasiholes (unoccupied electron states) which occur in the otherwise filled electron LL's as a result of recombination. The interacting states are shown schematically in Fig. 3 and consist of (i) one "hole" in the $N_{c}=0 \mathrm{LL}$ of the Fermi sea and (ii) one "hole" in the $N_{e}>1 \mathrm{LL}$, plus one LO phonon emitted in the PL process. Here $N_{e}>1$ is the parent LL of the resonating $N_{L}$ phonon satellite. The two states are coupled together by the Fröhlich hole-LO-phonon interaction. The resonance condition $\Delta N_{e} \hbar \omega_{c}=\hbar \omega_{\text {LO }}$ will be fulfilled in the magneto-PL of all systems where the electron Fermi energy $E_{F}$ is $\gtrsim \hbar \omega_{\text {LO }}$ such that the energy separation between filled LL's can be tuned through the energy region of $\hbar \omega_{\text {LO }}$.

In Fig. 4, the normalized relative intensities of the $N_{L}$ satellites $\left[N_{L}\right.$ intensity divided by the $N_{L}$ plus $(0,0)_{1}$ intensities] are plotted against the estimated unperturbed transition energy separations. The relative $N_{L}$ intensities of Fig. 4 are obtained from the observed peak intensities, after subtraction of the $(0,0)$, contribution, assuming constant PL linewidths with field. The accuracy is limited to approximately $\pm 30 \%$ in the relative intensities, particularly close to resonance because of the difficulty in separating the contribution from two closely overlapping lines.

Close to resonance, the satellite intensities are described quite well in terms of the hybridized $(0,0)_{1}$ and $N_{L}$ final

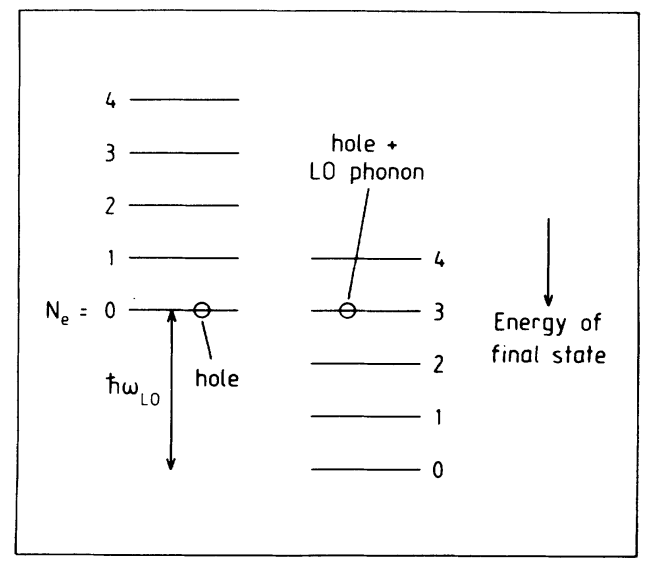

FIG. 3. Schematic diagram showing the resonating quasihole energy levels involved in the final states of the PL transitions. The final states without and with emission of an LO phonon are shown on the left- and right-hand sides of the figure, respectively.

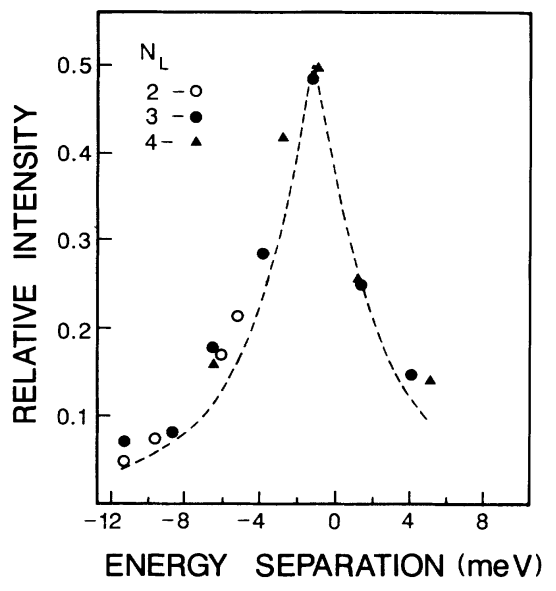

FIG. 4. Plot of $N_{l}$. satellite intensity divided by sum of $N_{L}$. and $(0,0)$, intensities as a function of unperturbed energy separation between the two transitions. The dashed curve is a fit to a model of two interacting states with mixing potential $\Delta E / 2=2.2$ $\mathrm{meV}$.

states, treated as a two-level coupled system. This is a marked simplification of RPC theory, ${ }^{8,20,21}$ but describes behavior similar to that predicted by perturbation theory close to resonance. ${ }^{8,20}$ Using the value $\Delta E / 2=2.2 \mathrm{meV}$ (from Fig. 2) for the electron-phonon mixing potential, the variation of intensities with energy separation, shown by the dashed curve in Fig. 4, is obtained. This provides a reasonable fit to the experimental points in Fig. 4, and demonstrates the expected exchange of oscillator strength between two interacting levels which anticross at resonance. $^{22}$

It is instructive to draw comparisons of the LL-LOphonon coupling phenomena observed in magneto-PL with previous studies in CR. ${ }^{2-6}$ In pure $2 \mathrm{D}$, the electronLO-phonon interaction is expected to be enhanced relative to $3 \mathrm{D}$, but due to the nonzero spatial extent of the electron wave function normal to the layers and to screening, the electron-phonon coupling strength in real doped heterojunctions (HJ's) (and QW's) is reduced to values close to or less than those observed in 3D. ${ }^{8-10,20}$ The most detailed studies of resonant polaron effects in GaAs$\mathrm{Ga}_{1-x} \mathrm{Al}_{x} \mathrm{As} \mathrm{HJ}$ 's have been carried out by Langerak et $a l .{ }^{5}$ These workers studied the cyclotron mass enhancements close to resonance for $n_{s}$ from 0.8 to $5.4 \times 10^{11}$ $\mathrm{cm}^{-2}$. The values for CR mass enhancements can be expressed in terms of the splitting $(\Delta E)$, assumed symmetric, between the upper and lower polaron branches at resonance. A very strong reduction of $\Delta E$ from $\sim 5.0$ $\mathrm{meV}$ at $3.4 \times 10^{11} \mathrm{~cm}^{-2}$, to about a factor of 2 smaller at $5.4 \times 10^{11} \mathrm{~cm}^{-2}$, was observed in Ref. 5 . The value of $\Delta E$ at $3.4 \times 10^{11} \mathrm{~cm}^{-2}$ is close to that found at $4 \times 10^{11} \mathrm{~cm}^{-2}$ by Sigg, Wyder, and Perenboom. ${ }^{6}$ At still higher densities $\left(9 \times 10^{11} \mathrm{~cm}^{-2}\right)$, Ziesmann, Heitmann, and Chang found no evidence for polaron coupling at resonance from $C R$ in a $200-\AA \operatorname{InAs} Q W .{ }^{4}$

These results for the magnitude of the RPC in CR with increasing $n_{s}$ are in strong contrast to the present PL results, where $\Delta E=4.4 \mathrm{meV}$ is observed for the $3_{L}-(0,0)$, 
resonance, at the much higher density of $1.6 \times 10^{12} \mathrm{~cm}^{-2}$ The contrast between magneto-PL (resonance with $N_{e}=3$ LL) and CR (resonance with $N_{e}=1 \mathrm{LL}$ ) is even greater when account is taken of the likely scaling of $\Delta E$ with Landau index. $\Delta E$ has been calculated to vary as $N_{e}^{-2 / 3}$ (Ref. 21) leading to predicted $\Delta E$ values $3^{2 / 3} \approx 2$ times smaller for magneto-PL in the present experiment (resonance with $N_{e}=3 \mathrm{LL}$ ) than for CR (resonance with $N_{e}=1 \mathrm{LL}$ ).

The very large strength of the resonant polaron interaction for the hole quasiparticles in PL, by comparison with $\mathrm{CR}$, can be understood qualitatively by consideration of the role of LL occupation effects. ${ }^{5,8-10}$ In CR such occupation effects are very important. The resonant coupling arises between the $N_{e}=1 \mathrm{LL}$ and the $N_{e}=0,1-\mathrm{LO}$ phonon state. As the $N_{e}=0$ population increases with $n_{s}$ (in Ref. 5, LL filling $v=0.67$ at $3.4 \times 10^{11} \mathrm{~cm}^{-2}, v=1$ at $5.4 \times 10^{11} \mathrm{~cm}^{-2}$ at resonance), the polaron interaction will be increasingly quenched, due to the occupation of the necessary scattering states in $N_{e}=0$. At complete filling, the resonant contribution to the CR mass enhancement is absent. ${ }^{10}$ Good agreement has been found in Ref. 5 with calculations in which the occupation effect is the dominant many-particle factor.

By contrast in magneto-PL such occupation effects are not important, since the scattering of the holes occurs be- tween otherwise filled LL's; all states within the resonating levels are available for the quasihole scattering since the hole levels are unoccupied. Coulomb screening alone acts to reduce the strength of the polaron interactions. ${ }^{23}$ Extrapolation of the predictions for $\Delta E$ for a $100-\AA \mathrm{QW}$ in Ref. 10 to $n_{s}=1.6 \times 10^{12} \mathrm{~cm}^{-2}$, including only the $n_{s}$ dependence of the screening (no occupation effects), leads to reasonable agreement ${ }^{24}$ with the value of $\Delta E=4.4 \mathrm{meV}$ observed in magneto-PL. However, a more detailed analysis of the present polaron phenomena clearly requires a theoretical treatment specifically directed towards the present case of hole-quasiparticle-LO-phonon coupling.

In conclusion, new resonant polaron coupling phenomena have been observed in the PL spectra of filled Landau levels in modulation-doped QW's. The results are interpreted in terms of hole-LO-phonon coupling in the final state of the Fermi sea. Such hole-quasiparticle-LOphonon coupling is expected to be a universal feature of the magneto-PL spectra of all QW's with electron Fermi energy equal to or greater than $\hbar \omega_{\text {LOO }}$.

We are grateful to L. Swierkowski for very helpful suggestions and to C.J.G.M. Langerak and R.J. Nicholas for informative discussions. One of us (P.E.S.) acknowledges financial support from RSRE.
'E. J. Johnson and D. M. Larsen, Phys. Rev. Lett. 16, 655 (1966).

${ }^{2}$ G. Lindemann, R. Lassnig, W. Seidenbusch, and E. Gornik, Phys. Rev. B 28, 4693 (1983).

${ }^{3}$ M. Horst, U. Merkt, and J. P. Kotthaus, Phys. Rev. Lett. 50, 754 (1983)

${ }^{4}$ M. Ziesmann, D. Heitmann, and L. L. Chang, Phys. Rev. B 35, 4541 (1987).

${ }^{5}$ C. J. G. M. Langerak, J. Singleton, P. J. van der Wel, J. A. A. J. Perenboom, D. J. Barnes, R. J. Nicholas, M. A. Hopkins, and C. T. Foxon, Phys. Rev. B 38, 13133 (1988).

${ }^{6}$ H. Sigg, P. Wyder, and J. A. A. J. Perenboom, Phys. Rev. B 31, 5253 (1985).

${ }^{7}$ RPC between LL's has also been studied recently in resonant tunneling structures. G. S. Boebinger, A. F. J. Levi, R. Schmitt-Rink, A. Passner, L. N. Pfeiffer, and K. W. West, Phys. Rev. Lett. 65, 235 (1990).

${ }^{8}$ D. M. Larsen, Phys. Rev. B 30, 4595 (1984).

${ }^{9}$ R. Lassnig, Surf. Sci. 170, 549 (1986).

${ }^{10}$ Xiaoguang Wu, F. M. Peeters, and J. T. Devreese, Phys. Rev. B 40, 4090 (1989); Phys. Status Solidi (b) 143, 581 (1987).

"Nonresonant satellites have been discussed by K. J. Nash, M. S. Skolnick, P. A. Claxton, and J. S. Roberts, Phys. Rev. B 39, 5558 (1989). Weak features in $B=0$ PL spectra were attributed to LO satellites of recombination near $E_{F}$ by C. Colvard, N. Nouri, H. Lee, and D. Ackley, Phys. Rev. B 39, 8033 (1989).

12P. B. Kirby, J. A. Constable, and R. S. Smith, Phys. Rev. B 40, 3013 (1989).

${ }^{13}$ M. S. Skolnick, D. M. Whittaker, P. E. Simmonds, T. A. Fisher, M. K. Saker, J. M. Rorison, P. B. Kirby, and C. R. H.
White, Phys. Rev. B 43, 7354 (1991).

${ }^{14}$ M. S. Skolnick, K. J. Nash, S. J. Bass, P. E. Simmonds, and M. J. Kane, Solid State Commun. 67, 637 (1988).

${ }^{15}$ E. D. Jones, S. K. Lyo, I. J. Fritze, J. F. Klem, J. E. Schirber, C. P. Tigges, and T. J. Drummond, Appl. Phys. Lett. 54, 2227 (1989).

${ }^{16}$ W. Chen, M. Fritze, A. V. Nurmikko, D. Ackley, C. Colvard, and H. Lee, Phys. Rev. Lett. 64, 2434 (1990).

${ }^{17}$ M. S. Skolnick, P. E. Simmonds, and T. A. Fisher, Phys. Rev. Lett. 66, 963 (1991); W. Chen, M. Fritze, and A. V. Nurmikko, Phys. Rev. Lett. 66, 964 (1991).

${ }^{18}$ P. G. Harper, J. W. Hodby, and R. A. Stradling, Rep. Prog. Phys. 36, I (1973)

${ }^{19}$ M. S. Skolnick and M. K. Saker (unpublished).

${ }^{20}$ S. Das Sarma and A. Madhukar, Phys. Rev. B 22, 2823 (1980); S. Das Sarma, ibid. 27, 2590 (1983).

${ }^{21}$ F. M. Peeters and J. T. Devreese, Phys. Rev. B 31, 3689 (1985).

${ }^{22}$ Such an exchange of oscillator strength at resonance, of course, is characteristic of any two-level coupled system, independent of the nature of the mixing potential. The good fit to the experimental results of Fig. 4 does not imply support for any specific model for the hole-LO-phonon coupling.

${ }^{23}$ Reference 10 shows that the reduction of $\Delta E$ by occupation effects is considerably larger than that due to Coulomb screening, for partially filled LL's.

${ }^{24}$ We estimate a value of $\Delta E=3.8 \mathrm{meV}$ at $n_{s}=1.6 \times 10^{12} \mathrm{~cm}^{-2}$ from the results of Ref. 10 with no occupation effects, after making allowance for the $N_{c}$ dependence of $\Delta E$ of $\Delta E \propto N_{c}^{-}-2 / 3$ (Ref. 21). In the absence of screening, the corresponding value would be $\Delta E \simeq 5.7 \mathrm{meV}$ (Ref. 10). 